\title{
Loss of Skeletal Muscle Mass and Poor Prognosis in Patients with ST-Segment Elevation Myocardial Infarction: Underling Mechanisms and Therapeutic Strategies
}

\author{
Kazunori Shimada ${ }^{1,2}$
}

${ }^{1}$ Department of Cardiovascular Medicine, Juntendo University Graduate School of Medicine, Tokyo, Japan

${ }^{2}$ Sportology Center, Juntendo University Graduate School of Medicine, Tokyo, Japan

Key words: Skeletal muscle mass, Sarcopenia, Prognosis, Myocardial infarction, Physical activity

\section{See article vol. 27: 1278-1287}

The terms of "muscle wasting," "sarcopenia," and "cachexia" are often overlapping and confusing. In 1860, Mauriac first used the term "cachexia" in his medical thesis, which is derived from the Greek words "kakós" for bad and "hexis" for condition ${ }^{1)}$. The term "sarcopenia" is described from the Greek words "sarx" for flesh and "penia" for loss, and it was first used by Rosenberg in 1989 to describe a primary loss of skeletal muscle and strength but not body weight ${ }^{2)}$. Muscle wasting and sarcopenia cannot be diagnosed simply weighting the patients. In 2010, the European Working Group on Sarcopenia in Older People (EWGSOP) proposed a diagnostic algorithm for sarcopenia, in which both muscle quantity and quality were fundamentally required to be evaluated ${ }^{3)}$. In 2014, the Asian Working Group for Sarcopenia (AWGS) defined sarcopenia as "age-related loss of muscle mass, with low muscle strength and/or physical performance," and proposed a diagnostic algorithm based on data derived from studies on Asian populations ${ }^{4)}$. The EWGSOP (EWGSOP2) and AWGS (AWGS 2019) updated the consensus in 2018 and 2019, respectively ${ }^{5,6)}$. According to AWGS 2019, the diagnosis of sarcopenia requires an evaluation of both muscle quantity and quality, and it defines individuals with a low muscle mass, muscle strength, and physical performance as having "severe sarcopenia" ${ }^{6)}$. Cachexia is a complex metabolic syndrome characterized by severe and involuntary loss of muscle mass with or without fat mass wasting. The cutoff value of weight for diagnosing cachexia still remains a topic of debate ${ }^{7,8)}$.
The loss of muscle mass, including muscle wasting, sarcopenia, and cachexia, is associated with aging and various diseases. Sarcopenia is associated with clinical outcomes such as increased risk of physical limitations, slowness, falls, fractures, frailty, and mortality $^{6,7)}$. Cachexia is associated with not only cancer, but also inflammatory conditions such as chronic obstructive pulmonary disease, heart failure, chronic kidney disease, acquired immunodeficiency syndrome, and sepsis ${ }^{7-9)}$.

In this issue of the Journal of Atherosclerosis Thrombosis, Sato et al. have reported novel aspects of decreased skeletal muscle mass and poor outcomes in 387 patients with ST-segment elevation myocardial infarction (STEMI) ${ }^{10)}$. The skeletal muscle mass of the study participants was measured using dual-energy $\mathrm{X}$-ray absorptiometry. The subjects were divided into low- and high- appendicular skeletal mass index (ASMI; appendicular skeletal muscle mass divided by height squared) groups using the first quartile 50 of ASMI $\left(\leq 6.64 \mathrm{~kg} / \mathrm{m}^{2}\right.$ for men and $\leq 5.06 \mathrm{~kg} / \mathrm{m}^{2}$ for women). At baseline, the age and prevalence of complex coronary lesion and Killip classification $\geq 2$ were higher. The left ventricular ejection fraction levels were lower in the low-ASMI group than those in the high-ASMI group. During a median follow-up of 33 months, the primary composite events, including allcause death, nonfatal myocardial infarction, nonfatal ischemic stroke, hospitalization for heart failure, and unplanned revascularization, were significantly higher in the low-ASMI group than in the high-ASMI group $(24.7 \%$ vs $13.4 \%)$. After adjustment of the values for clinical backgrounds, a low ASMI value was found to be independently associated with a high risk of pri-

Address for correspondence: Kazunori Shimada, Department of Cardiovascular Medicine, Juntendo University Graduate School of Medicine, 2-1-1 Hongo Bunkyo-ku, Tokyo 113-8421, Japan E-mail: shimakaz@juntendo.ac.jp.

Received: May 13, 2020 Accepted for publication: May 17, 2020

Copyright@2020 Japan Atherosclerosis Society

This article is distributed under the terms of the latest version of CC BY-NC-SA defined by the Creative Commons Attribution License. 
mary composite events (hazard ratio, 2.06) ${ }^{10)}$.

As mentioned by the authors, this study is the first to present important findings regarding the significant association between decreased skeletal muscle mass and poor outcomes in patients with STEMI ${ }^{10)}$. Interestingly, a lower cutoff value may be better for predicting the primary composite events in male patients with STEMI compared with the AWGS criteria, which was developed based on studies on young Asian adults. A recent study has reported that the cross-sectional area of the skeletal muscle at the first lumbar vertebral level revealed by computed tomography is a powerful predictor of adverse outcomes; this study included 475 patients undergoing percutaneous coronary intervention ${ }^{11)}$. Therefore, further studies are essential for determining the clinical significance and optimal cutoff value of skeletal muscle mass especially in patients with cardiovascular diseases.

The skeletal muscle is one of the fundamental organs enabling locomotion and respiration and contains the largest protein pool in the whole body. Therefore, imbalances between protein synthesis and degradation underlie the loss of skeletal muscle mass ${ }^{7,8,12)}$. Numerous factors contribute to muscle wasting, including sympathetic nervous system, hormone balance, the renin-angiotensin-aldosterone system, insulin-like growth factor, proinflammatory cytokines, reactive oxygen species production, glucocorticoids, and members of the transforming growth factor- $\beta$ family such as myostatin ${ }^{7,8,12)}$ Protein synthesis is mainly regulated by the phosphoinositide 3-kinaseserine/threonine-protein kinase-mammalian target of rapamycin-p70S6K (PI3K-AKT-mTOR-p70S6K) signaling pathway. In contrast, protein degradation is regulated by three major pathways: activation of the ubiquitin-proteasome system, including muscle RING finger-1 (MuRF-1) and MAFbx/atrogin-1 (atrogin-1); autophagy; and apoptosis mediated by caspase signaling. Notably, the PI3K-AKT-mTOR pathway inhibits Forkhead box $\mathrm{O}$ protein (FOXO) and prevents the activation of myostatin-decapentaplegic homolog 2 (SMAD2) pathways ${ }^{7,8,12)}$ (Fig. 1). Furthermore, we have recently reported that oxidative stress is associated with mitochondrial dysfunction and muscle wasting using an aging mouse model and NADPH oxidase activation is associated with angiotensin II-induced muscle wasting ${ }^{13,14)}$.

Numerous interventions such as nutritional and pharmacological support have been used to manage muscle wasting associated with sarcopenia and cachexia. Essential amino acids, high-calorific/proteinrich nutritional supplements, vitamin $\mathrm{D}$, growth hormone, ghrelin, testosterone replacement therapy, $\beta_{2}-$ adrenegic agents, and omega 3 polyunsaturated fatty acids might be beneficial to specific patients with primary muscle wasting and cachexia ${ }^{7-9,15,16)}$. Thalidomide, an anti-inflammatory agent, and anti-myostatin neutralizing antibodies or soluble decoy receptors have been recently reported to increase the muscle mass and strength, however, their clinical benefits are still be discussed ${ }^{7-9,15,16)}$. In contrast, exercise is an alternative clinical intervention that may be the most beneficial to patients with reduced skeletal muscle mass and strength. Physical inactivity is not only a major contributor to muscle wasting in cachexia and sarcopenia but also an important risk factor for the development of cardiovascular diseases ${ }^{17}$. Exercise training and physical activity result in numerous molecular alterations in skeletal muscles (Fig. 1), improved quality of life, reduced hospitalization frequency, and prolonged survival $^{7-9,15-17)}$. Therefore, exercise should be considered a cornerstone in the treatment of skeletal muscle wasting even in patients with cachexia and sarcopenia.

In conclusion, this study clearly demonstrates that a decreased skeletal muscle mass is significantly associated with poor clinical outcomes in patients with STEMI. Currently, the evaluation of skeletal muscle mass and strength and assessment of nutritional status and physical activity are insufficient in the clinical setting. In addition, an early and appropriate approach is necessary before muscle wasting, sarcopenia, and cachexia manifest. Hence, further basic investigations and clinical studies are required in this regard.

\section{Conflicts of Interests}

None.

\section{Funding}

High Technology Research Center Grant, Strategic Research Foundation at Private Universities and KAKENHI (17K01470, 19K11374) from the Ministry of Education, Culture, Sports, Science and Technology of Japan.

\section{References}

1) C. Mauriac: Essai sur les maladies de cœur: de la mort subite dans l'insuffisance das valvules sigmoides de l'aorte, L. 1860; Leclerc, Paris

2) Rosenberg IH: Summary comments: epidemiological and methodological problems in determining nutritional status of older persons. Am J Clin Nutr, 1989; 50: 12311233

3) Cruz-Jentoft AJ, Baeyens JP, Bauer JM, Boirie Y, Cederholm T, Landi F, Martin FC, Michel JP, Rolland Y, Schneider SM, Topinková E, Vandewoude M, Zamboni M; European Working Group on Sarcopenia in Older People. 


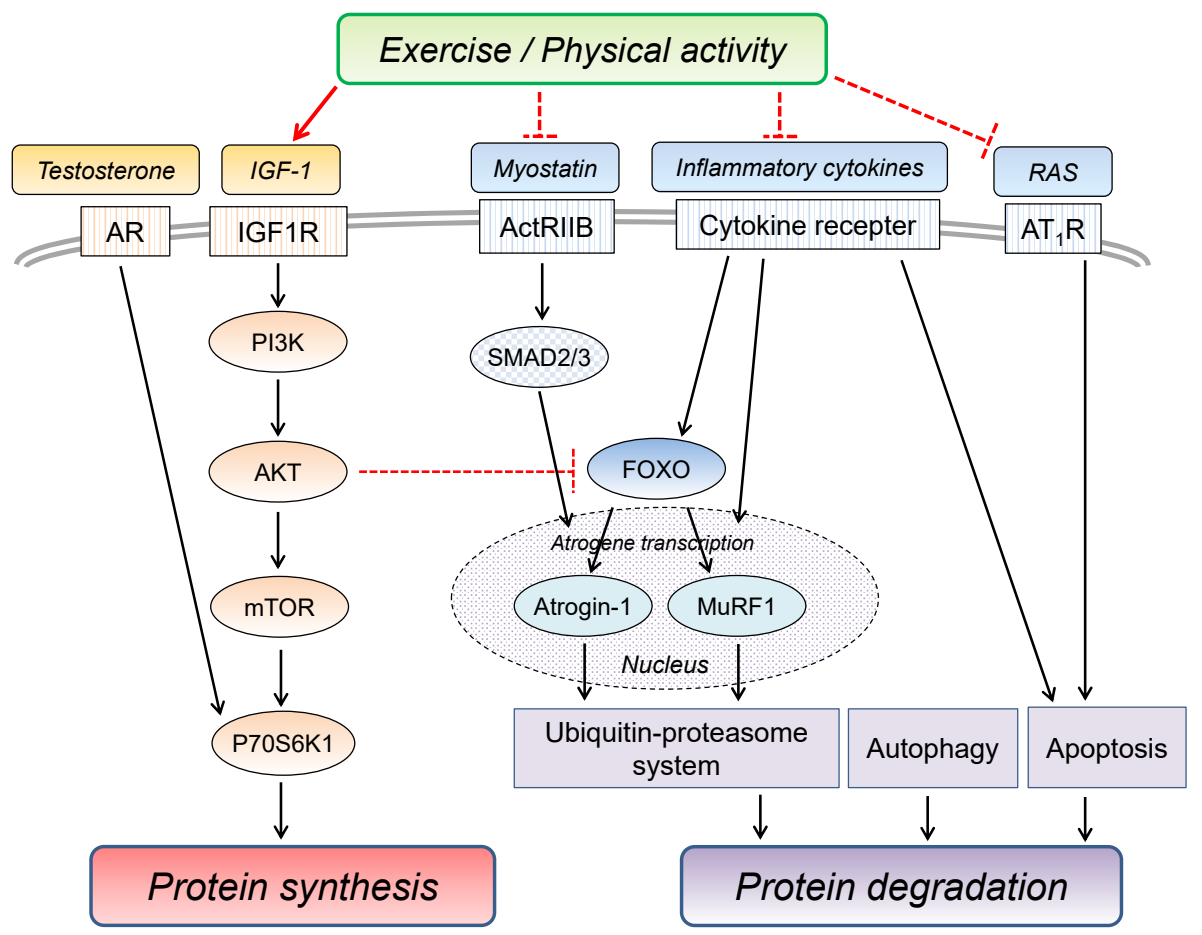

Fig. 1. Protein synthesis and degradation of skeletal muscle

AR, adrenergic receptor; IGF, insulin-like growth factor; IGF1R, IGF-1 receptor; PI3K, phosphoinositide 3-kinase; mTOR, mammalian target of rapamycin; p70S6K, p70 ribosomal S6 kinase; ActRIIB, activin receptor IIb; AKT, serine/threonine-protein kinase; FOXO, Forkhead box O protein (FOXO); atrogin-1, MAFbx/ atrogin-1; MuRF-1, muscle RING finger-1; RAS, renin-angiotensin system; AT1R, angiotensin II type 1 receptor.

Sarcopenia: European consensus on definition and diagnosis: Report of the European Working Group on Sarcopenia in Older People. Age Ageing, 2010; 39: 412-423

4) Chen LK, Liu LK, Woo J, Assantachai P, Auyeung TW, Bahyah KS, Chou MY, Chen LY, Hsu PS, Krairit O, Lee JS, Lee WJ, Lee Y, Liang CK, Limpawattana P, Lin CS, Peng LN, Satake S, Suzuki T, Won CW, Wu CH, Wu SN, Zhang T, Zeng P, Akishita M, Arai H: Sarcopenia in Asia: consensus report of the Asian Working Group for Sarcopenia. J Am Med Dir Assoc, 2014; 15 :95-101

5) Cruz-Jentoft AJ, Bahat G, Bauer J, Boirie Y, Bruyère O, Cederholm T, Cooper C, Landi F, Rolland Y, Sayer AA, Schneider SM, Sieber CC, Topinkova E, Vandewoude M, Visser M, Zamboni M; Writing Group for the European Working Group on Sarcopenia in Older People 2 (EWGSOP2), and the Extended Group for EWGSOP2: Sarcopenia: revised European consensus on definition and diagnosis. Age Ageing, 2019; 48: 16-31

6) Chen LK, Woo J, Assantachai P, Auyeung TW, Chou MY, Iijima K, Jang HC, Kang L, Kim M, Kim S, Kojima T, Kuzuya M, Lee JSW, Lee SY, Lee WJ, Lee Y, Liang CK, Lim JY, Lim WS, Peng LN, Sugimoto K, Tanaka T, Won CW, Yamada M, Zhang T, Akishita M, Arai H. Asian Working Group for Sarcopenia: 2019 Consensus Update on Sarcopenia Diagnosis and Treatment. J Am Med Dir Assoc, 2020; 21: 300-307

7) Bowen TS, Schuler G, Adams V: Skeletal muscle wasting in cachexia and sarcopenia: molecular pathophysiology and impact of exercise training. J Cachexia Sarcopenia Muscle, 2015; 6: 197-207

8) von Haehling S, Ebner N, Dos Santos MR, Springer J, Anker SD: Muscle wasting and cachexia in heart failure: mechanisms and therapies. Nat Rev Cardiol, 2017; 14: 323-341

9) Suzuki T, Palus $S$, Springer J: Skeletal muscle wasting in chronic heart failure. ESC Heart Fail, 2018; 5: 10991107

10) Sato R, Akiyama E, Konishi M, Matsuzawa Y, Suzuki H, Kawashima C, Kimura Y, Okada K, Maejima N, Iwahashi N, Hibi K, Kosuge M, Ebina T, von Haehling S, Anker SD, Tamura K, Kimura K: Decreased appendicular skeletal muscle mass is associated with poor outcomes after STsegment elevation myocardial infarction. J Atheroscler Thromb, 2020; 27: 1278-1287

11) Kang DO, Park SY, Choi BG, Na JO, Choi CU, Kim EJ, Rha SW, Park CG, Hong SJ, Seo HS: Prognostic impact of low skeletal muscle mass on major adverse cardiovascular events in coronary artery disease: A propensity scorematched analysis of a single center all-comer cohort. J Clin Med, 2019; 8: E712

12) Kinugawa $S$, Takada $S$, Matsushima $S$, Okita $K$, Tsutsui $\mathrm{H}$ : Skeletal Muscle Abnormalities in Heart Failure. Int Heart J, 2015; 56: 475-484

13) Kadoguchi T, Shimada K, Koide H, Miyazaki T, Shiozawa 
T, Takahashi S, Aikawa T, Ouchi S, Kitamura K, Sugita Y, Hamad AS, Kunimoto M, Sato-Okabayashi Y, Akita K, Isoda K, Daida $\mathrm{H}$ : Possible role of NADPH oxidase 4 in angiotensin II-induced muscle wasting in mice. Front Physiol, 2018; 9: 340

14) Kadoguchi T, Shimada K, Miyazaki T, Kitamura K, Kunimoto M, Aikawa T, Sugita Y, Ouchi S, Shiozawa T, Yokoyama-Nishitani M, Fukao K, Miyosawa K, Isoda K, Daida $\mathrm{H}$ : Promotion of oxidative stress is associated with mitochondrial dysfunction and muscle atrophy in aging mice. Geriatr Gerontol Int, 2020; 20: 78-84

15) Saitoh M, Ishida J, Doehner W, von Haehling S, Anker
MS, Coats AJS, Anker SD, Springer J: Sarcopenia, cachexia, and muscle performance in heart failure: Review update 2016. Int J Cardiol, 2017; 238: 5-11

16) Arai H, Wakabayashi $H$, Yoshimura Y, Yamada M, Kim H, Harada A: Chapter 4 Treatment of sarcopenia. Geriatr Gerontol Int, 2018; 18 Suppl 1: 28-44

17) Shimada K, Nishitani-Yokoyama M, Takahashi T, Daida $\mathrm{H}$ : Physical activity and long-term prognosis in patients with stable coronary artery disease: How often, how intense, and how long? Eur J Prev Cardiol, 2020; 27: $422-425$ 